\title{
Optimal sizing, scheduling and shift policy of the grinding section of a ceramic tile plant
}

\author{
Belmiro P.M. Duarte ${ }^{\mathrm{a}, \mathrm{b}, *}$, Lino O. Santos ${ }^{\mathrm{b}}$, Jorge S. Mariano ${ }^{\mathrm{a}}$ \\ a Department of Chemical Engineering, ISEC, Polytechnic Institute of Coimbra, R. Pedro Nunes, 3030-199 Coimbra, Portugal \\ ${ }^{\mathrm{b}}$ GEPSI-PSE Group, CIEPQPF, Department of Chemical Engineering, University of Coimbra, Pólo II, R. Slvio Lima, 3030-790 Coimbra, Portugal
}

\section{A R T I C L E I N F O}

Keywords:

Sizing

Scheduling

Resource task network

Mixed-integer linear programming

Ceramic industry

\begin{abstract}
A B S T R A C T
This paper addresses the optimal design of the grinding section of a ceramic tile plant operating in a cyclic mode with the units (mills) following a batch sequence. The optimal design problem of this single product plant is formulated with a fixed time horizon of one week, corresponding to one cycle of production, and using a discrete-time resource task network (RTN) process representation. The size of the individual units is restricted to discrete values, and the plant operates with a set of limited resources (workforce and equipment). The goal is to determine the optimal number and size of the mills to install in the grinding section, the corresponding production schedule, and shift policy. This problem involves labor/semi-labor intensive (LI/SLI) units with a depreciation cost of the same order as that of the operation cost. The optimal design of the grinding section comprises the trade-off between these two costs. The resulting optimization formulation is of the form of a mixed integer linear programming (MILP) problem, solved using a branch and bound solver (CPLEX 9.0.2). The optimal solution is analyzed for various ceramic tile productions and different shift policies.
\end{abstract}

\section{Scope and Purpose}

This paper addresses an optimal design case study of the grinding section of a ceramic tile plant with respect to the net capacity to install, the operation scheduling and the shift policy to implement. A mathematical programming model is formulated based on the resource task network (RTN) framework representation. The problem is solved using a branch and bound algorithm. The main goal of this work is to apply optimal design/scheduling general tools to real problems commonly found in the ceramic industry sector, a particular case of labor intensive plants. The application of these methodologies to this case study demonstrates as well the importance of adopting suitable optimization strategies for plant design in order to improve the economical performance of the production lines.

(c) 2008 Elsevier Ltd. All rights reserved.

\section{Introduction and motivation}

The planning and scheduling in the process industry has received considerable attention from the research community in the last decades. From the industrial point of view, Grossmann [1] observes that enterprise-wide optimization has become a major goal in the process industries due to the increasing pressures for remaining competitive in the global marketplace. In an increasingly competitive market, adequate process planning and scheduling is

* Corresponding author at: Department of Chemical Engineering, ISEC, Polytechnic Institute of Coimbra, R. Pedro Nunes, 3030-199 Coimbra, Portugal. Tel.: +351239790200

E-mail addresses: bduarte@isec.pt (B.P.M. Duarte),

lino@eq.uc.pt (L.O. Santos), jmariano@isec.pt (J.S. Mariano). essential to achieve significant economical benefits and to keep the plant in business. The category of problems in this area may include features such as multipurpose and multiproduct batch plants, combined cyclic material flows, multi-stage, batch and campaign production, and different storage and transference policies. Recent overviews describing the main features and current state of the art of planning and scheduling problems in the chemical process industry can be found in the works of Méndez et al. [2], Floudas and Lin [3], and Kallrath [4]. Early mathematical programming formulations for planning and scheduling process problems are based on a discrete-time representation. However, discrete-time formulations may require a large number of time slots to represent accurately a problem. This in turn requires a large number of binary variables associated to each discrete-time interval, leading to problems of higher dimensionality, and often numerically intractable. The adoption of a 
continuous time representation overcame these difficulties, and at the same time allowed to solve problems of higher dimensionality and complexity. For more details on the advantages and limitations of these approaches see for instance the discussion in the reviews by Floudas and Lin [3] and Méndez et al. [2].

The integration of the plant scheduling in the early stage of the plant design is essential to improve the economical goals. Therefore, the design of new processes or plants must take into consideration the interplay with the planning and scheduling aspects of the production. This trend motivated the formulation of even more complex optimization problems in an attempt to determine simultaneously the optimal plant sizing and scheduling. Several approaches have been reported in the literature to model and solve this class of problems. See for instance the reviews in Castro et al. [5] and in Floudas and Lin [3]. Yeh and Reklaitis [6] proposed a two stage approach for the design of single product batch/semicontinuous plants. The design problem is partitioned in two subproblems: the plant network synthesis subproblem and the sizing subproblem. These problems are solved by adopting an evolutionary strategy, and using an approximate method. Concerning the optimal integrated design of biochemical processes, Samsatli and Shah [7] also adopt a two-stage design approach. The processing times, rates and conditions for the batch tasks are determined in the first stage, whereas the scheduling of the plant operation is determined in the second stage.

In general, the mathematical formulations for simultaneous plant synthesis, sizing and scheduling can yield problems of the form of mixed integer non-linear programming (MINLP). However, these nonlinear representations can be simplified to mixed integer linear programming (MILP) problems when the size of the equipment is restricted to discrete values [8]. This assumption allows to address even more complex design models and systems. To handle the complexity associated with the representation of chemical processes in a systematic fashion, Kondili et al. [9] proposed a state-task network (STN) representation. This representation considers two types of nodes: states and tasks. State nodes represent feeds, intermediate and final products. On the other hand, task nodes represent the processing operations which transform material from one or more input states to one or more output states [9]. This suitable approach to represent the process was extended by Pantelides [10], resulting in a resource task network (RTN) representation. The RTN process representation can be viewed as an advanced version of the STN. The major difference between them is that the STN treats equipment resources implicitly, while the RTN treats them explicitly [5]. It involves two types of nodes: resources and tasks. Here, resource nodes represent all entities that describe the process, such as raw-materials and products, processing and storage equipment, utilities (workforce, energy), and the equipment condition or state (clean, dirty, empty, filled). All these formulations provide general tools to handle complex processes and focus on algorithms, taking advantage of the development of robust and fast optimization solvers. Several simultaneous design and scheduling problems using either STN or RTN representations, in the form of either discrete or continuous-time formulations, are reported in the literature. For instance, Barbosa-Póvoa and Macchietto [11] developed a formulation with a STN process representation to solve simultaneously the problem of the optimal plant equipment design, including the determination of their associated connections, and the associated production schedule. BarbosaPóvoa and Pantelides [12] use a discrete-time RTN representation to model the design problem of multipurpose plants, taking into account the trade-offs between capital costs, revenues and operational flexibility. Lin and Floudas [13] address the problem of integrated design, synthesis and scheduling of multipurpose batch plants using a continuous-time STN representation. Maravelias and Grossmann [14] proposed a novel algorithm for the minimization of the makespan of multipurpose batch plants using the STN formulation.
Castro et al. [5] present a general mathematical formulation for the simultaneous design and scheduling of multipurpose plants based on a continuous-time RTN representation.

The case study addressed in this work was motivated by a Portuguese ceramic factory planning to install a new facility to produce ceramic tile. Typically, ceramic tile plants feature continuous operating units such as furnaces and involve some degree of labor/semilabor intensive (LI/SLI) batch units as well, such as mills. The Portuguese ceramic company was particularly interested in the optimal design of the grinding section. The grinding section is a single product plant of LI/SLI type, with a set of ball mills operating in batch mode. The optimal design of LI/SLI plants must address the trade-off between the operation costs and the installed equipment depreciation costs. Resources such as workforce and energy very often account for an important part of the overall plant cost. Thus, the cost of the resources required to operate the plant may interact significantly with the plant design and/or synthesis. Hence, the equipment cost based objective function in typical sizing problems is replaced by a function that considers both the operation and depreciation costs of the equipment to install. Since these two costs depend on each other, the resulting optimal structure comprises the optimal design, the resources allocation policy, and the corresponding optimal schedule.

In this case study, the plant operates in a cyclic mode with a fixed horizon of one week. The grinding section is designed with mills of discrete capacity, available in the market with four different net capacities. The mills are loaded through conveyors under the supervision of operators, and their operation is energy intensive. The energy usage is defined in accordance with the commercialization policy practiced by the energy supplier. The objective of this single product plant optimal design problem is the determination of: (i) the number and net capacity of the mill units to be installed to satisfy a given demand, taking into account the discrete capacities of the mill units available in the market; (ii) the optimal plant schedule taking into account the workforce and equipment resources constraints; (iii) the optimal shift policy.

The paper is organized as follows. Section 2 introduces the formulation of the optimal design and scheduling problem of the grinding section of the ceramic tile plant, based on the discrete-time RTN process representation. Section 3 describes the problem of the optimal sizing and scheduling-Problem I-assuming that there is only one worker available to operate the entire grinding section. This formulation is extended in Section 4 to determine the optimal shift policy-Problem II-, where up to five operators are available to run the grinding section. Finally, concluding remarks are given in Section 5.

\section{RTN process representation}

The design and scheduling problem in this case study involves individual batch units, and their net capacity is restricted to discrete values. The grinding section produces one single product, and its operation is cyclic with a fixed time horizon of 1 week. These characteristics fit on a discrete-time formulation since it leads to a mild optimization problem in terms of dimensionality and complexity. The discrete-time feature allows to handle problems where time dependent resource constraints are set a priori, such as the workforce. This class of problems can be handled either with a discretetime STN or a discrete-time RTN representation. These formulations lead to different model constraints (the RTN representation treats the equipment resources explicitly) and different computational performances [5]. Recent results for scheduling problems favor the RTN representation [15].

The cyclic scheduling problem is modelled using a discrete-time RTN framework [10]. The RTN framework stands on the representation of the process by a set of topological entities comprising two 
types of nodes: tasks and resources. Tasks represent operations that change the state of the product or piece of equipment. Resources are entities necessary to promote state changes. The time horizon of one production cycle, $H$, is discretized into $T$ time slots (Fig. 1 ) bounded by event points or time events $t, t=0, \ldots, T$. This set of time events, $t \in \mathscr{T}$, defines the time instants where process changes may occur, such as resources allocation and state changes. All time slots have the same time duration of $\Delta \delta$ units of time. The value of $\Delta \delta$ is selected to be equal to the time required to complete the fastest task. The $t_{\text {slot }}$ th time slot, $t_{\text {slot }}=t, t \geqslant 1$, starts at the event point $t-1$ and ends at the event point $t$ (Fig. 1). The absolute time at which the production cycle is initialized is $\delta_{0}$, and the absolute times at which an event may occur are $\delta=\delta_{0}+t \Delta \delta$, for $t=0, \ldots, T$.

A smaller time slot duration $\Delta \delta$ (higher number of event points) leads to a better accuracy of the model representation. However, the problem complexity increases with the decrease of $\Delta \delta$, and the model dimension is proportional to $H / \Delta \delta$. In general, accurate model representations can be obtained if the discretization interval is made equal to the duration of the shortest task [9]. This allows to trade off the model dimension with the accuracy, and consequently to reduce the computational effort.

A discrete representation of the time horizon is adopted to formulate the problem because the time availability of the workforce

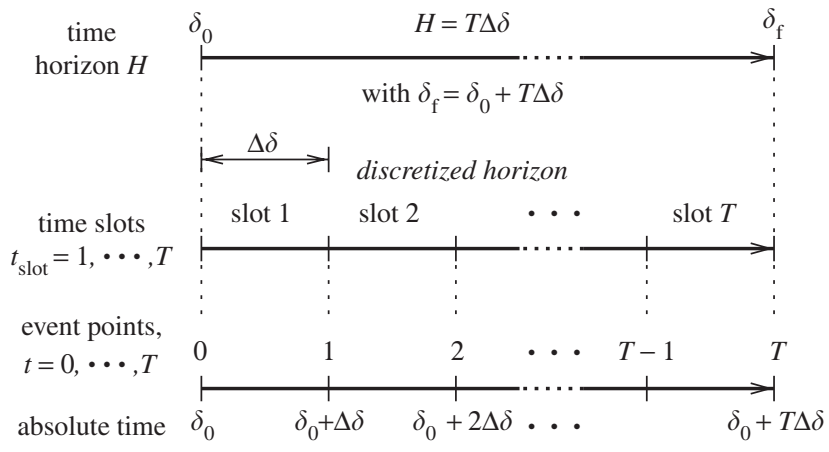

Fig. 1. Time horizon discretization. Definition of time slots, time events or event points, and the corresponding absolute time. resources, and the time dependent cost of the energy is fixed a priori. The inclusion of the workforce availability constraints and the energy cost in the model formulation is straightforward after setting the absolute time at which the production cycle is initialized $\left(\delta_{0}\right)$ and the time horizon $(H)$ of the scheduling problem. The production scheduling must satisfy labor contracts enforced by the ceramic industry sector, and take advantage as much as possible of the lowest energy prices proposed by the energy supplier.

The resulting combinatorial formulation stands on the assumption that the plant topology is only partially known a priori. Although the sequence of operations of each piece of equipment is known, the number of pieces of equipment that are needed to satisfy the demand is not known a priori. The problem is formulated under the assumption that there are no constraints in the quantity of raw material used to feed the grinding section. After the grinding operation the clay is immediately feed to the formation units to produce ceramic tiles, which are later processed in continuous furnaces.

Table 1

Definition of the elements of the production/consumption matrices of the states and resources, $\omega_{k, s, \theta}$ and $\mu_{k, r, \theta}$, respectively

\begin{tabular}{|c|c|}
\hline $\begin{array}{l}\text { Task } k=1, \text { feeding, with a duration } \tau_{1}=1 \\
\theta=0,1\end{array}$ & $\begin{array}{l}\text { Task } k=3, \text { emptying, with } \tau_{3}=1 \\
\theta=0,1\end{array}$ \\
\hline $\begin{array}{l}\text { State } s=1 \text { (empty): } \omega_{1,1,0}=-1 ; \omega_{1,1,1}=0 \\
\text { State } s=2 \text { (filled): } \omega_{1,2,0}=0 ; \omega_{1,2,1}=+1 \\
\text { State } s=3 \text { (ground clay): } \omega_{1,3, \theta}=0, \theta=0,1 \\
\text { Resource } r=1 \text { (operator): } \mu_{1,1,0}=-1 ; \mu_{1,1,1}=+1 \\
\text { Resource } r=2 \text { (energy): } \mu_{1,2, \theta}=0, \theta=0,1 \\
\text { Resource } r=3 \text { (mill): } \mu_{1,3,0}=-1 ; \mu_{1,3,1}=+1 \\
\text { Task } k=2 \text {, grinding, with a duration } \tau_{2}=13 \\
\theta=0, \ldots, 13\end{array}$ & $\begin{array}{l}\omega_{1,1,0}=+1 ; \omega_{1,1,1}=0 \\
\omega_{1,2, \theta}=0, \theta=0,1 \\
\omega_{1,3,0}=-1 ; \omega_{1,3,1}=0 \\
\mu_{1,1,0}=-1 ; \mu_{1,1,1}=+1 \\
\mu_{1,2, \theta}=0, \theta=0,1 \\
\mu_{1,3,0}=-1 ; \mu_{1,3,1}=+1\end{array}$ \\
\hline $\begin{array}{l}\text { State } s=1 \text { (empty): } \omega_{2,1, \theta}=0, \theta=0, \ldots, 13 \\
\text { State } s=2 \text { (filled): } \omega_{2,2,0}=-1 ; \omega_{2,2, \theta}=0, \theta=1, \\
\text { State } s=3 \text { (ground clay): } \omega_{2,3, \theta}=0, \theta=0, \ldots, 12 ; \\
\text { Resource } r=1 \text { (operator): } \mu_{2,1, \theta}=0, \theta=0, \ldots, 13 \\
\text { Resource } r=2 \text { (energy): } \mu_{2,2,0}=-1 ; \mu_{2,2, \theta}=0, \theta \\
\text { Resource } r=3 \text { (mill): } \mu_{2,3,0}=-1 ; \mu_{2,3, \theta}=0, \theta=\end{array}$ & $\begin{array}{l}\ldots, 13 \\
\omega_{2,3,13}=+1 \\
=1, \ldots, 12 ; \mu_{2,2,13}=+1 \\
1, \ldots, 12 ; \mu_{2,3,13}=+1\end{array}$ \\
\hline
\end{tabular}

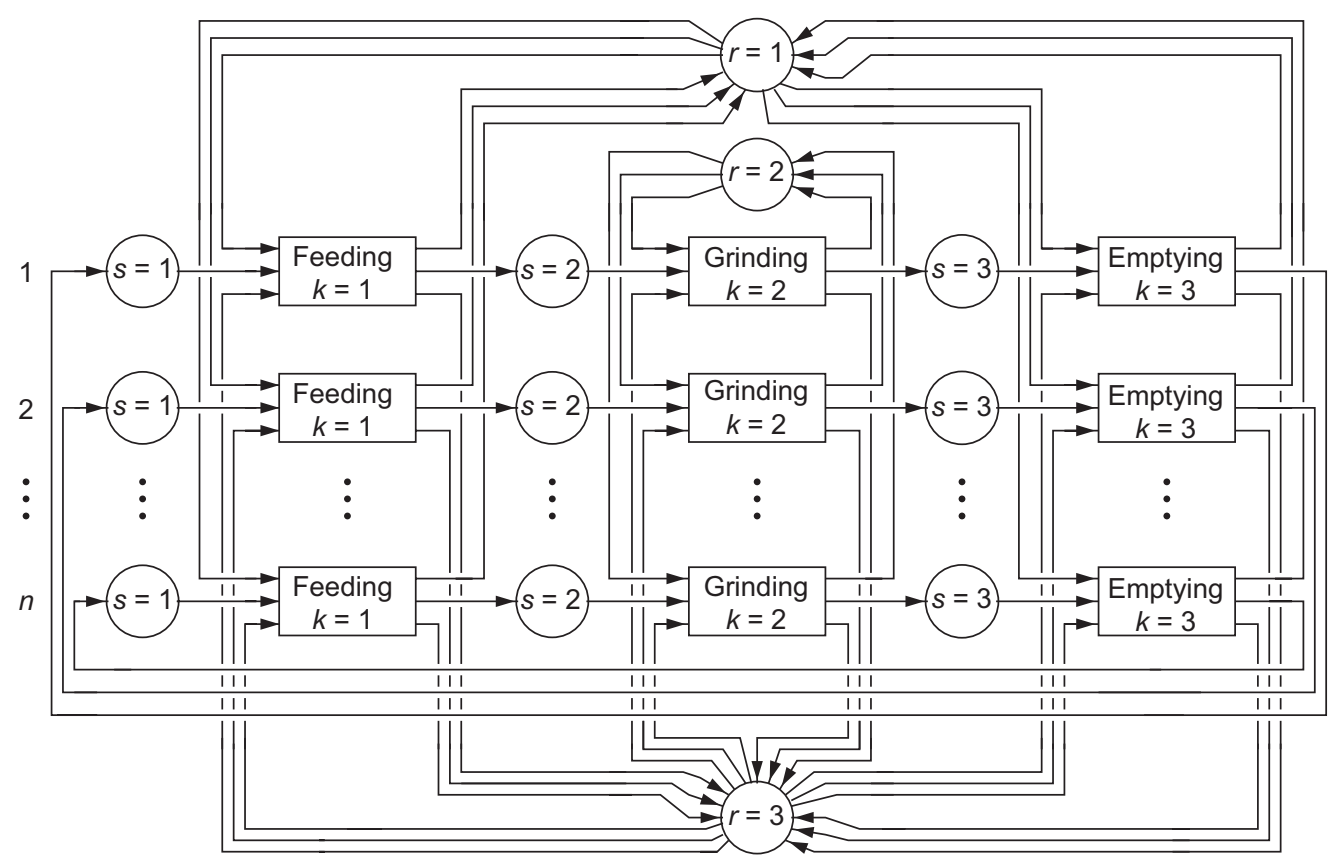

Fig. 2. Resource task network representation of the grinding section with $n$ mills. 


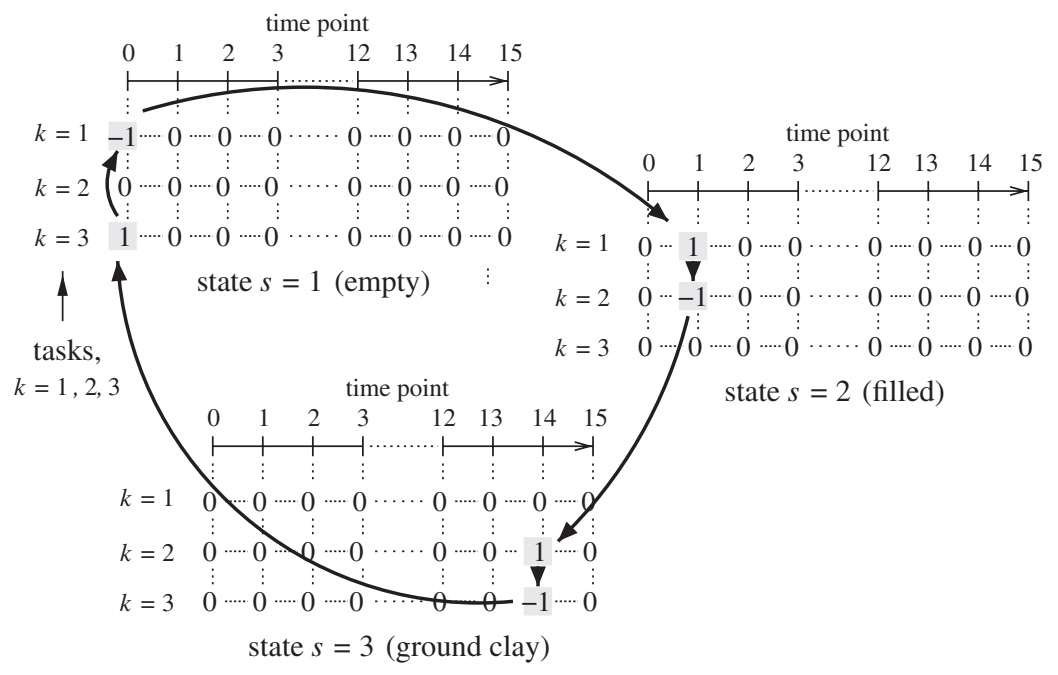

Fig. 3. Characterization of the status of states during one batch performed in $15 \mathrm{~h}$.

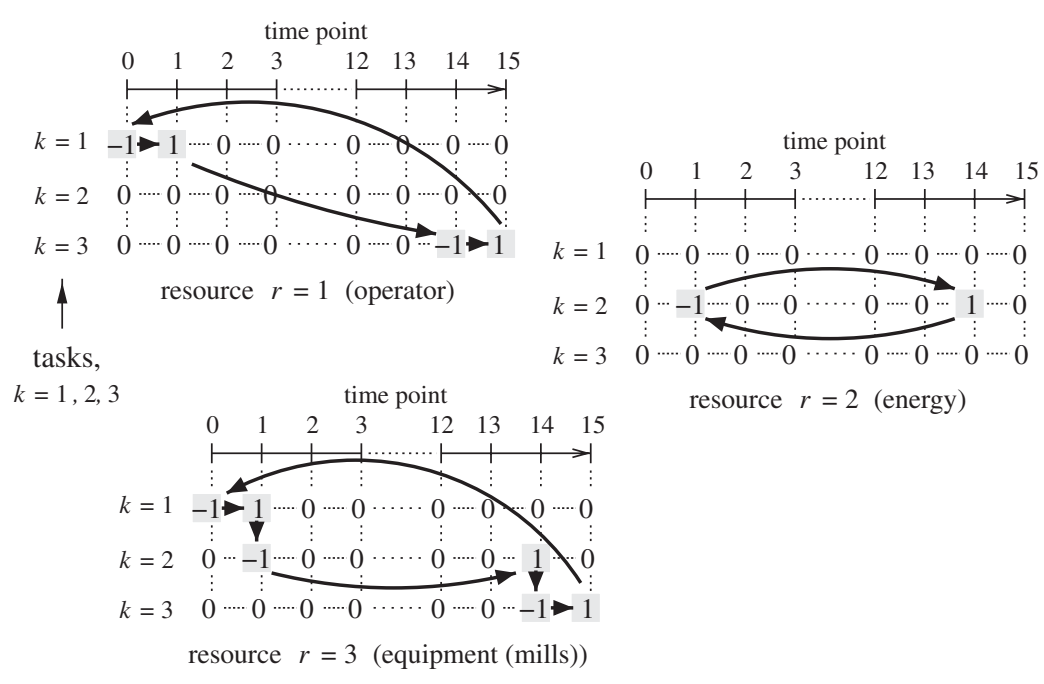

Fig. 4. Characterization of the status of resources during one batch performed in $15 \mathrm{~h}$.

The production demand is defined in terms of square meters of ceramic tile per week.

Fig. 2 summarizes the RTN process representation of the grinding section with a number of $n$ mills. Task nodes are denoted by rectangles whereas both states and resources nodes are denoted by circles $[10,16]$. The grinding section runs in batch mode and it involves a sequence of three tasks, $k=1,2,3$. The typical cycle of a batch associated to a given mill can be described as follows:

- $k=1$ : feed the mill with raw material. This task changes the state of the mill unit from empty $(s=1)$ to filled $(s=2)$, and needs one operator (resource $r=1$ ) to feed the mill. Each mill is filled using a different feed line. This avoids the need to address feeding lines schedule. This task has a time duration $\Omega_{1}=1 \mathrm{~h}$.

- $k=2$ : grind the material filled. This task changes the state of the raw material filled to ground clay $(s=3)$, and requires energy (resource $r=2$ ). The time duration of this task is $\Omega_{2}=13 \mathrm{~h}$.

- $k=3$ : release the ground clay. Here the mill state is changed from filled with ground clay $(s=3)$ to empty $(s=1)$, and the corresponding mill unit is made ready for a new load. This task requires also an operator (resource $r=1$ ) to discharge and clean the mill. The time duration of this task is $\Omega_{3}=1 \mathrm{~h}$.
Therefore it takes at least $15 \mathrm{~h}$ to complete one batch (feeding, grinding, and emptying) per mill. The time duration of the fastest tasks (feeding, $k=1$, and emptying, $k=3$ ) is of $1 \mathrm{~h}$. Hence, the time horizon of the cycle of production $H$ (Fig. 1) must be discretized using time intervals of $\Delta \delta=1 \mathrm{~h}$. As explained in Section 3, the time horizon $H$ for this problem is fixed to one week, $168 \mathrm{~h}$, corresponding to 169 time events, $t=0, \ldots, 168$.

Remark that all tasks require the operation of a mill (resource $r=3$ ). Also, to satisfy a given production demand it may be necessary to install more than one piece of equipment. Hence, the installed $n$ mills must be numbered appropriately in the problem formulation, $m=1, \ldots, n$ (Fig. 2). In the RTN representation described in Fig. 2, it is assumed that the consumption of energy to perform tasks 1 and 3 (feeding and emptying, respectively) is not significant in comparison to the energy necessary for the grinding task. It is noteworthy to mention that the states of the RTN representation $(s=1,2,3)$ characterize as well the state of the mill units over the production cycle.

Each task $k$ has a fixed time duration expressed in terms of the number of time intervals, defined by $\tau_{k}=\operatorname{int}\left(\Omega_{k} / \Delta \delta\right)$. The time duration of each task is used to define the so-called production/consumption matrices of the states and resources. These matrices are used to characterize the status of the states and 
resources during the batch, with respect to each task, and over the entire time horizon of the production cycle. Resources and states are produced and consumed at a finite set of discrete times during the execution of a given task [10]. An integer binary variable, $N_{m, i, k, t}$, is introduced to characterize the operation of task $k$ at time $t$ related to the mill or grinding unit $m$ of net capacity $i . N_{m, i, k, t}$ defines the extent of task $k$, and determines the demands that it places on the resources and states. Following the lines of the description in [10], the amount of resource $r$ (or state $s$ ) produced at the time point $\theta$ relative to the start of task $k$ at time point $t$ is assumed to be related to the extent through a relationship of the form $\mu_{k, r, \theta} N_{m, i, k, t-\theta}$ (or $\omega_{k, s, \theta} N_{m, i, k, t-\theta}$ ), where $\mu_{k, r, \theta}$ and $\omega_{k, r, \theta}, \theta=0, \ldots, \tau_{k}$, are known constants. A negative value of $\mu_{k, r, \theta}$ or $\omega_{k, s, \theta}$ indicates consumption of the resource or state, respectively. A positive value indicates production. For a more detailed description see [10]. The values of $\mu_{k, r, \theta}$ and $\omega_{k, S, \theta}$ for this case study are given in Table 1.

Figs. 3 and 4 illustrate the use of the constants defined in Table 1 to characterize one batch operation of a mill under the assumption there are no dead times between tasks. Thus, the minimum time to complete one batch (feeding, grinding, and emptying) is $15 \mathrm{~h}$. However, in practice a batch may take longer to be completed because of the resource constraints. The elements of the production/consumption matrices, $\omega_{k, s, \theta}$ and $\mu_{k, r, \theta}$, are used in Figs. 3 and 4 to define the status of the states and resources at each time point $t_{\mathrm{b}}$ of the batch, $t_{\mathrm{b}}=0,1, \ldots, 15$, with respect to each task $k$. For instance, in Fig. 3, regarding the feeding task $(k=1)$, at the time point $t_{\mathrm{b}}=0$ the state empty $(s=1)$ is consumed, $\omega_{1,1,0}=-1$. One hour later $\left(t_{\mathrm{b}}=1\right)$, the state filled $(s=2)$ is produced, $\omega_{1,2,1}=+1$. To perform the feeding task the resource operator $(r=1)$ (Fig. 4$)$ is consumed at $t_{\mathrm{b}}=0, \mu_{1,1,0}=-1$, and produced (released) at $t_{\mathrm{b}}=1$, $\mu_{1,1,1}=+1$. This task involves the allocation (consumption) of the resource mill $(r=3)$ at $t_{\mathrm{b}}=0$, and its release (production) at $t_{\mathrm{b}}=1$, $\mu_{1,3,0}=-1$ and $\mu_{1,3,1}=+1$, respectively. Both energy and mill resources $\left(r=2\right.$ and 3 ) are consumed at $t_{\mathrm{b}}=1$ (relative time point $\theta=0$ ), $\mu_{2,2,0}=-1$ and $\mu_{2,3,0}=-1$, to perform the grinding task $(k=2)$, and produced at time point $t_{\mathrm{b}}=14$ (relative time point $\theta=13$ ), $\mu_{2,2,13}=+1$ and $\mu_{2,3,13}=+1$. The arrows in Figs. 3 and 4 illustrate the cyclic nature of the states and resources status changes as described in Table 1.

The mills are operated as units with intermediate storage capacity because after the feed task they can be programmed to start later the grinding operation. Therefore, after the end of the grinding operation the ground clay can wait inside the mill until being discharged. Therefore, depending on the availability of the workforce resource, the time to run one batch may be longer than $15 \mathrm{~h}$. This transference policy provides the opportunity to optimally schedule the units operation taking into account both the labor work availability and the energy cost.

\section{Optimal sizing and scheduling}

The optimal sizing and scheduling problem described in this section-denoted by Problem I-is formulated assuming that there is only one single operator available to operate the entire grinding section. The working period of time stipulated for the ceramic industry sector is from $08: 00$ to $12: 00$ and from 14:00 to 18:00. Also, it is assumed that the operator can only feed or discharge one mill at a time. The operator might be allocated to perform other tasks required by the process operation in the remaining time, but those are not addressed in the present case study. The cost of the labor work is based on the average salary in the Portuguese ceramic industry sector ( $€ 161.50$ per worker and week). The length of the time horizon of the production cycle $H$ is of $168 \mathrm{~h}$, starting on Monday at 00:00. Note that the workforce is not available during the weekend. Since the grinding mills can operate during the weekend, the final task (release of the ground clay) may be accomplished only when the operator is back on Monday at 08:00. The absolute initial time of the production cycle, $\delta_{0}$, is set to 00:00 of Monday, and as explained in Section 2, the time slot duration is $\Delta \delta=1 \mathrm{~h}$ (Fig. 1). This gives origin to a discretized horizon with 169 event points.

The proposed problem formulation is not concerned with the start-up or shut-down stages of the plant operation. It is focused on finding the optimal plant design and scheduling for one weekly cycle of production. In normal operating conditions the same production scheduling is repeated week after week. Therefore, it is possible that one or more tasks start in one cycle and finish into the next one. Shah et al. [17] introduced the concept of task wrap-around to address the problem of tasks that start within the cycle of interest and finish into the next cycle. The conceptual idea standing in this approach is to displace the tasks extending beyond the cycle duration to the beginning of the cycle, thus producing the so-called wrap-around effect. To take this into account in the problem constraints formulation, a wrap-around operator is formulated as follows [17]:

$\sigma(t)= \begin{cases}t & \text { if } t \geqslant 0 \\ t+T & \text { if } t<0\end{cases}$

where $T$ is the fixed number of time intervals of the production cycle (Fig. 1), and $t=0, \ldots, T$, with $T=168$. For example, $\sigma(-1)=\sigma(167)=167$. Further details on this concept can be found in [17], as well as in various applications to periodic scheduling problems reported in the literature, e.g., [18-21].

The indices, sets, variables, and parameters associated with Problem I formulation are described as follows:

Indices

$i \quad$ unit size

$k \quad$ task

$m \quad$ unit number

$r$ resource

$s \quad$ state

$t \quad$ time event or event point

$\theta \quad$ time index

Sets

$\mathscr{I} \quad\{i$ : set of all net capacities of units $\}$

$\mathscr{K} \quad\{k:$ set of all tasks $\}$

$\mathscr{M} \quad\{m:$ set of all unit numbers of net capacity $i\}$

$\mathscr{R} \quad\{r$ : set of all resources $\}$

$\mathscr{S} \quad\{s:$ set of all states $\}$

$\mathscr{T} \quad\{t \geqslant 0:$ set of all time events $\}$

$\mathscr{T}^{S} \quad\{t>0$ : set of all time slots $\}$

$\mathscr{T}_{r}^{u} \quad\left\{t_{r}\right.$ : set of all time events at which resource $r$ is not available $\}$

Continuous variables

$P \quad$ weekly tile production demand

Z total cost

\section{Binary variables}

$N_{m, i, k, t}$ assigns the task $k$ at unit $m$ of net capacity $i$ at time event $t$

$Q_{m, i, t}$ assigns the availability of mill unit $m$ of net capacity $i$ at time event $t$

$R_{r, t} \quad$ assigns the availability of resource $r$ at time event $t$

$S_{m, i, s, t}$ assigns the state $s$ at unit $m$ of net capacity $i$ at time event $t$

$y_{m, i}$ assigns the unit number $m$ to the net capacity $i$

\section{Parameters}

$C_{i} \quad$ depreciation cost of the unit of net capacity $i$

$C_{\mathrm{R} r, t}$ cost of resource $r$ at time $t$

$\mathrm{H}$ time horizon

$k^{\mathrm{f}} \quad$ task that releases the final product

$V_{i} \quad$ net capacity of unit $i$ 
W weight of clay necessary to make one square meter of ceramic tile

$\Delta \delta \quad$ time slot duration

$\mu_{k, r, t}$ consumption/production of resource $r$ in task $k$ at event point $t$

$\rho$ density of the clay material

$\sigma(\bullet)$ wrap-around operator

$\tau_{k} \quad$ number of time intervals of task $k$, with $\tau_{k}=\operatorname{int}\left(\Omega_{k} / \Delta \delta\right)$

$\Omega_{k} \quad$ duration of task $k$

$\omega_{k, s, t}$ consumption/production of state $s$ in task $k$ at event point $t$

The size of the set $\mathscr{M}$ is defined by using a heuristic rule. Its size is equal to $m_{\max }$, the number of mills of largest net capacity ( $V_{\max }$ ) required to achieve the desired weekly production rate if only a single batch is processed per day in each unit, 5 days a week. $m_{\max }$ is obtained from the following ceiling function:

$m_{\max }=\left\lceil\frac{P}{5} \cdot \frac{W}{0.5 V_{\max } \rho}\right\rceil$

where $V_{\max }=\max _{i \in \mathscr{I}}\left\{V_{i}\right\}$. From Table 3, it follows that $V_{\max }=$ $50000 \mathrm{~L}$. In Eq. (2), $V_{\max }$ is multiplied by a factor of 0.5 . This factor is due to an equipment manufacturer recommendation regarding the operation of a mill: to resume normal and safe operation the mill can be feed up to a maximum of $50 \%$ of its net capacity. $P$ is the weekly tile production demand. The density of the clay obtained from the grinding process is $\rho=1.625 \mathrm{~kg} / \mathrm{L}$. To obtain one square meter of ceramic tile requires on average $17 \mathrm{~kg}$ of ground clay, $W=17 \mathrm{~kg} / \mathrm{m}^{2}$. For example, if $P=42000 \mathrm{~m}^{2}$, it follows that $m_{\max }=4$. One emphasizes that $m_{\max }$ stands for the maximal number of units of net capacity $V_{\max }$ that can be assigned in order to satisfy the production demand $P$. Obviously, if one considers the available set of net capacities, the number of mills ( $n$ in Fig. 2 ) to satisfy $P$ may be greater than $m_{\max }$. For instance, the design and scheduling problem for $P=42000 \mathrm{~m}^{2}$ is formulated assuming that up to 16 mills can be installed, four of each net capacity $i$ (Table 3 ).

Hence, Eq. (2) sets an upper bound on the number of mills of maximal capacity required to satisfy the production demand. This condition enforces as much as possible the selection of mills with the greatest net capacity, and consequently leads to design solutions with the least number of mills. This is understood under the assumption that the installation and operation costs would be the same for every type of mill, which is not the case (see Table 3). As described next, these different costs are obviously also taken into account in the problem formulation.

The optimal design problem is based on the minimization of an objective cost function $Z$ comprising both the operation and depreciation costs of the equipment. The model has the form of a MILP problem, and is stated as follows:

$$
\min \quad Z=\sum_{m \in \mathscr{M}} \sum_{i \in \mathscr{I}} y_{m, i} C_{i}+\sum_{r \in\{1,2\}} \sum_{t \in \mathscr{T}^{S}}\left(1-R_{r, t}\right) C_{\mathrm{R}, r, t}
$$

s.t. $S_{m, i, s, t}=S_{m, i, s, \sigma(t-1)}+\sum_{k \in \mathscr{K}} \sum_{\theta=0}^{\tau_{k}} w_{k, s, \theta} N_{m, i, k, \sigma(t-\theta)}$,

$\forall m \in \mathscr{M}, i \in \mathscr{I}, s \in \mathscr{S}, t \in \mathscr{T}^{s}$

$$
R_{r, t}=R_{r, \sigma(t-1)}+\sum_{m \in \mathscr{M}} \sum_{i \in \mathscr{I}} \sum_{k \in \mathscr{K}} \sum_{\theta=0}^{\tau_{k}} u_{k, r, \theta} N_{m, i, k, \sigma(t-\theta)},
$$$$
r \in\{1,2\}, \forall t \in \mathscr{T}^{S}
$$

$$
Q_{m, i, t}=Q_{m, i, \sigma(t-1)}+\sum_{k \in \mathscr{K}} \sum_{\theta=0}^{\tau_{k}} u_{k, r, \theta} N_{m, i, k, \sigma(t-\theta)},
$$$$
r=3, \forall m \in \mathscr{M}, i \in \mathscr{I}, t \in \mathscr{T}^{S}
$$

Table 2

Energy cost policy

\begin{tabular}{llll}
\hline Period & & Day & $€ / \mathrm{kWh}$ \\
\hline 1 & $09: 00-11: 00,19: 00-21: 00$ & Mon.-Fri. & 0.2162 \\
2 & $07: 00-09: 00,11: 00-19: 00,21: 00-24: 00$ & Mon.-Fri. & 0.0945 \\
3 & $00: 00-07: 00$ & Mon.Fri. & 0.0481 \\
4 & $09: 00-13: 00,19: 00-22: 00$ & Saturday & 0.0945 \\
5 & $00: 00-9: 00,13: 00-19: 00,22: 00-24: 00$ & Saturday & 0.0481 \\
6 & $00: 00-24: 00$ & Sunday & 0.0481 \\
\hline
\end{tabular}

Table 3

Characteristics of the mill units available in the market

\begin{tabular}{llll}
\hline Type of mill & Net capacity, L & $\begin{array}{l}\text { Depreciation cost, } \\
€ / \text { week }\end{array}$ & $\begin{array}{l}\text { Energy consumption, } \\
\text { kWh }\end{array}$ \\
\hline M1 & 50000 & 1084.44 & 105.0 \\
M2 & 35000 & 867.55 & 92.5 \\
M3 & 25000 & 759.11 & 80.0 \\
M4 & 15000 & 672.35 & 67.5 \\
\hline
\end{tabular}

$$
\begin{aligned}
& Q_{m, i, t} \leqslant y_{m, i}, \quad \forall m \in \mathscr{M}, \quad i \in \mathscr{I}, \quad t \in \mathscr{T}^{S} \\
& \sum_{i \in \mathscr{I}} y_{m, i}=1, \quad \forall m \in \mathscr{M} \\
& \sum_{m \in \mathscr{M}} \sum_{i \in \mathscr{I}} \sum_{t \in \mathscr{T}^{S}} N_{m, i, k^{\mathrm{f}}, t} V_{i} \geqslant \frac{P W}{0.5 \rho} \\
& y_{m, i} V_{i} \leqslant y_{m-1, i} V_{i}, \quad \forall i \in \mathscr{I}, \quad m>1 \\
& R_{r, t}=1, \quad \forall r \in \mathscr{R}, \quad t \in \mathscr{T}_{r}^{u} \\
& y, N, S, R, Q \in\{0,1\}
\end{aligned}
$$

Eq. (3) represents the total cost accounting for both the equipment depreciation costs and the cost of the resources (workforce and energy) needed to run the plant. Eqs. (4)-(6) represent the states and the resources balances at each event point. Eq. (4) accounts for the states availability, and Eq. (5) represents the workforce and energy (resources $r=1,2$ ) balances. Eq. (6) establishes the availability of the mill units (resource $r=3$ ). The equality constraints Eqs. (4)-(6) are formulated with the wrap-around operator $\sigma(\bullet)$ to account for the possibility of one or more tasks that start in one cycle of production and finish into the next one. This also guarantees that the status of the states and of the resources are the same at the beginning $(t=0)$ and at the end $(t=168)$ of the production cycle.

Eq. (7) represents the allocation of unit $m$ of discrete net capacity $i$ whenever such an equipment resource is employed to carry out a task. Eq. (8) aims at reducing the effort required during the process of solving the MILP problem with the branch and bound solver, by stating that each unit can have only one discrete capacity $i \in \mathscr{I}$. Eq. (9) is a constraint in order to satisfy the weekly tile production demand $P$. As explained previously for Eq. (2), in practice only $50 \%$ of the net capacity can be used.

Eq. (10) enforces that the process units needed to satisfy the tile production demand are numbered in a decreasing order of their capacities. The purpose of this condition is to reduce the degeneracy of the minimization problem solution. Eq. (11) states that resource $r$ is not available at the time events of subset $\mathscr{T}_{r}^{u}$. Eq. (11) contains information on a priori known resources unavailability over the entire horizon $H$. For instance, it is known a priori that the workforce resource $(r=1)$ is unavailable $\left(R_{1, t}=1\right)$ for time events within the weekend period, and time events outside the time intervals from 08:00 to $12: 00$ and from $14: 00$ to $18: 00$. It is assumed that energy $(r=2)$ and equipment $(r=3)$ resources are always available over the entire time horizon $H$. Hence, in these two cases constraint (11) does not apply because $\mathscr{T}_{r}^{u}=\emptyset, r=2,3$.

The energy is subject to a price policy established by the energy supplier, and depends on the period of the day and of the week (Table 2). Table 3 presents the four sizes of mills (M1, M2, M3, M4) available in the market, their discrete net capacity, depreciation cost, and 
Table 4

Optimal solutions of the Problem I for various weekly ceramic tile productions, $P$

\begin{tabular}{rllllll}
\hline$P / \mathrm{m}^{2}$ & M1 & M2 & M3 & M4 & $Z^{*} / €$ & CPU time $/ \mathrm{s}^{\mathrm{a}}$ \\
\hline 7000 & - & 1 & - & - & 1543.77 & 3.8 \\
14000 & 1 & - & - & 1 & 2715.20 & 9.5 \\
21000 & 2 & - & - & - & 3372.18 & 3.1 \\
28000 & 1 & 2 & - & - & 4594.77 & 15.4 \\
35000 & 3 & - & - & - & 5167.66 & 16.4 \\
42000 & 3 & 1 & - & - & 6464.73 & 151.4 \\
49000 & \multicolumn{2}{l}{ Infeasible solution } & & & & \\
\hline
\end{tabular}

a On a Windows XP Intel Core 2 Duo $1.83 \mathrm{GHz}$ computer.

energy consumption. The depreciation cost is determined assuming a life time period of 9.5 years for the equipment [22].

To summarize, Problem I involves three types of resources (equipment units, energy and workforce), three tasks, and three states. The energy resource is not subject to any time constraint availability. Its cost is included in the objective function as one of the components of the operation costs, such that the resulting optimal schedule takes into account the energy supplier commercialization policy. Regarding the equipment resources, its cost is accounted by the first term of the objective function (3).

The resulting MILP problem is solved for several ceramic tile demand production scenarios (Table 4), using the solver GAMS/CPLEX 9.0.2 [23], with a relative tolerance of $10^{-3}$. For tile productions equal or higher than $49000 \mathrm{~m}^{2}$ per week the solution becomes infeasible because the only existing operator cannot carry out more than four charges and discharges per day. These tasks altogether require the $8 \mathrm{~h}$ of daily work stipulated for the ceramic industry sector. It is pointless to install more mills since the limiting factor is the workforce resources availability. The CPU time (Table 4 ) exhibits a non-regular behavior as the ceramic tile production demand increases. This behavior can be explained as follows. A given production target may require a combination of units/net capacities such that the overall installed grinding capacity is oversized, and therefore the mills are operated in a sub-intensive mode. This feature can lead to degenerated solutions, where some batches and tasks can be assigned to different time events without significant variation of the objective function. The optimal task assignment is then partially dictated by the energy cost, which has a lower impact on the overall cost. This causes an increase in the number of feasible solutions to search, with a consequent increase of the overall CPU time.

\subsection{Schedule for a tile production demand of $42000 \mathrm{~m}^{2}$ per week}

The optimal schedule of the grinding section for a ceramic tile demand scenario of $42000 \mathrm{~m}^{2}$ per week is illustrated in Fig. 5. It describes how the activities (tasks) associated to each mill are distributed over the time horizon of the production cycle, from $\delta_{0}=0$ to $\delta_{\mathrm{f}}=168 \mathrm{~h}$. The optimal solution suggests the installation of four ( $n=4$ in Fig. 2 ) mills (Table 4 ): three mills of class M1 (with a net capacity of $50000 \mathrm{~L}$; mills 1, 2 and 3 in Fig. 5), and one mill of class M2 (with a net capacity of $35000 \mathrm{~L}$; mill 4 in Fig. 5). Thus the total installed net capacity is of $185000 \mathrm{~L}$. As explained previously, in practice only half of the net capacity can be used, that is $92500 \mathrm{~L}$. To obtain one square meter of ceramic tile requires on average $17 \mathrm{~kg}$ of ground clay with $\rho=1.625 \mathrm{~kg} / \mathrm{L}$. Because of the working period of the operator, the mills can be loaded and/or discharged only from Monday to Friday. Also, because the grinding task takes $13 \mathrm{~h}$, each mill can only perform one batch per day, and during only 5 days a week. Therefore the maximum tile production that can be achieved with this set of mills is of $44210 \mathrm{~m}^{2}$ per week, which is greater than the weekly production demand $\left(42000 \mathrm{~m}^{2}\right)$. This explains why the mill of class M2 (mill 4 in Fig. 5) is used in a sub-intensive way, performing only four batches per week. On the other hand, the tree mills of class M1 are used in an intensive way as they process five batches each. The optimal solution also shows that task $k=2$ (grinding) is carried out mainly during the night to take profit of the lowest energy costs (Period 3 in Table 2). As expected, the feed and discharge tasks occur during the operator working period of time. The last four grinding tasks of the production cycle occur during the weekend to fully take advantage of the lowest cost of energy from saturday 22:00 to monday 07:00. Here, the solution of the problem is not unique as these four grinding tasks can start at any time between saturday 22:00 and sunday 18:00 without affecting the final production cost.

\section{Optimal sizing, scheduling and shift policy}

The problem introduced in Section 3 with only one operator is modified to handle workforce resources with up to five workers. Here, the problem formulation is extended in order to incorporate the decision about the optimal shift policy, and is denoted by Problem II. There are three possible alternative shift policies to be implemented. They are set according to the Portuguese Labor Contract work policy for the ceramic industry sector:

- $p=1$, shift policy P1: one worker, 5 days a week, with daily working periods from 08:00 to 12:00 and from 14:00 to 18:00;

- $p=2$, shift policy P2: two workers, 5 days a week, with daily working periods from 07:00 to 15:00 and from 14:00 to 22:00;

- $p=3$, shift policy P3: five workers, 7 days a week, with a daily working period of $24 \mathrm{~h}$.

The problem formulation with shift policies is simplified by taking into account only the total number of hours of work available for the production cycle. The goal is to assign hours of work to the needs of the plant production without distinction on which worker is being assigned to each task. This aspect of the scheduling problem is not addressed here because it would require additional information on the other workforce plant activities. According to the Portuguese Labor Contract work policy, the individual weekly workload is of $40 \mathrm{~h}$. Hence, shift policy P2 provides $80 \mathrm{~h}$ of workforce a week whereas shift policy P3 provides $200 \mathrm{~h}$.

The indices, sets, variables, and parameters associated with Problem II are the same as those presented for the formulation of Problem I, excepting for the new index $p$ (shift policy), set $\mathscr{P},\{p$ : set of all shift policies $\}$, and $\mathscr{T}_{p, r}^{u},\left\{t_{p, r}\right.$ : set of all time events at which resource is not available for the shift policy $p$. There is an additional binary variable as well, to assign the shift policy, $x_{p}$. The binary variables and parameters with index $p$ are defined as follows:

Binary variables

$N_{p, m, i, k, t}$ assigns task $k$ to unit $m$ of net capacity $i$ at time event $t$, with shift policy $p$

$Q_{p, m, i, t}$ assigns the availability of mill $m$ of net capacity $i$ at time event $t$, with shift policy $p$

$R_{p, r, t} \quad$ assigns the availability of resource $r$ at time event $t$, with shift policy $p$

$S_{p, m, i, s, t}$ assigns the state $s$ at unit $m$ of net capacity $i$ at time event $t$, with shift policy $p$

$y_{p, m, i}$ assigns the unit $m$ to net capacity $i$, with shift policy $p$

$x_{p} \quad$ assigns the shift policy $p$

The MILP formulation of Problem II is as follows:

$\begin{aligned} \min Z= & \sum_{p \in \mathscr{P}} \sum_{m \in \mathscr{M}} \sum_{i \in \mathscr{I}} y_{p, m, i} C_{i} \\ & +\sum_{p \in \mathscr{P}} \sum_{r \in\{1,2\}} \sum_{t \in \mathscr{T}^{S}}\left(1-R_{p, r, t}\right) C_{\mathrm{R}, r, t}\end{aligned}$ 


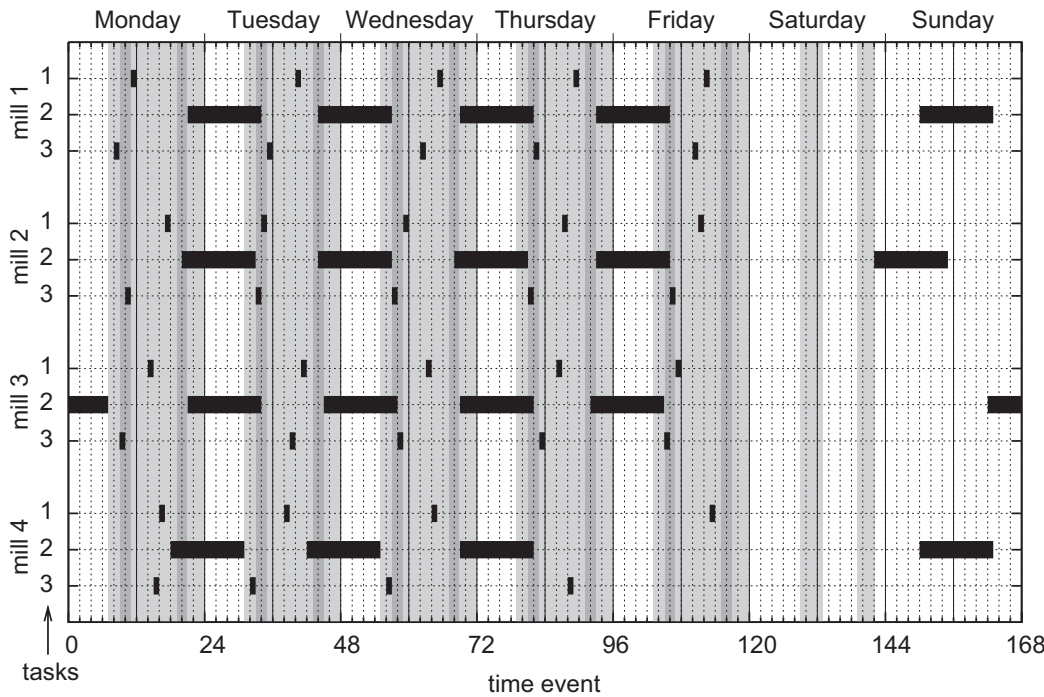

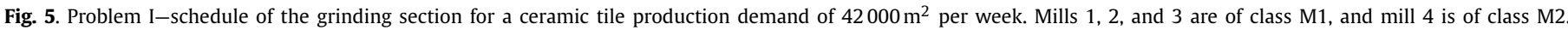
The white, light gray, and dark gray areas correspond to the energy cost of $0.0481,0.0945$, and $€ 0.2161$ per $\mathrm{kW}$ h, respectively.

$$
\begin{aligned}
& \text { s.t. } \quad S_{p, m, i, s, t}=S_{p, m, i, s, \sigma(t-1)}+\sum_{k \in \mathscr{K}} \sum_{\theta=0}^{\tau_{k}} w_{k, s, \theta} N_{p, m, i, k, \sigma(t-\theta)} \text {, } \\
& \forall p \in \mathscr{P}, m \in \mathscr{M}, \quad i \in \mathscr{I}, \quad s \in \mathscr{S}, t \in \mathscr{T}^{s} \\
& R_{p, r, t}=R_{p, r, \sigma(t-1)}+\sum_{m \in \mathscr{M}} \sum_{i \in \mathscr{I}} \sum_{k \in \mathscr{K}} \sum_{\theta=0}^{\tau_{k}} u_{k, r, \theta} N_{p, m, i, k, \sigma(t-\theta)}, \\
& r \in\{1,2\}, \quad \forall p \in \mathscr{P}, \quad t \in \mathscr{T}^{S} \\
& Q_{p, m, i, t}=Q_{p, m, i, \sigma(t-1)}+\sum_{k \in \mathscr{K}} \sum_{\theta=0}^{\tau_{k}} u_{k, r, \theta} N_{p, m, i, k, \sigma(t-\theta)}, \\
& r=3, \forall p \in \mathscr{P}, m \in \mathscr{M}, i \in \mathscr{I}, t \in \mathscr{T}^{S} \\
& Q_{p, m, i, t} \leqslant y_{p, m, i}, \quad \forall p \in \mathscr{P}, \quad m \in \mathscr{M}, \quad i \in \mathscr{I}, t \in \mathscr{T}^{S} \\
& \sum_{p \in \mathscr{P}} \sum_{i \in \mathscr{I}} y_{p, m, i} \leqslant 1, \quad \forall m \in \mathscr{M} \\
& \sum_{m \in \mathscr{M}} \sum_{i \in \mathscr{I}} \sum_{t \in \mathscr{T}^{S}} N_{p, m, i, k^{\mathrm{f}}, t} V_{i} \geqslant \frac{P W}{0.5 \rho}, \quad \forall p \in \mathscr{P} \\
& \sum_{i \in \mathscr{I}} y_{p, m, i} V_{i} \leqslant \sum_{i \in \mathscr{I}} y_{p, m-1, i} V_{i}, \quad \forall m>1, \quad p \in \mathscr{P} \\
& R_{p, r, t}=1, \quad \forall p \in \mathscr{P}, \quad r \in \mathscr{R}, \quad t \in \mathscr{T}_{p, r}^{u} \\
& \sum_{i \in \mathscr{I}} y_{p, m, i} \leqslant x_{p}, \quad \forall p \in \mathscr{P}, \quad m \in \mathscr{M} \\
& \sum_{p \in \mathscr{P}} x_{p}=1 \\
& y, N, S, R, Q, x_{p} \in\{0,1\}
\end{aligned}
$$

Eqs. (12)-(20) generalize Eqs. (3)-(11) for the set of shift policies considered, $p \in \mathscr{P}$. Eq. (21) states that the shift policy $p$ is assigned whenever there is at least one unit under operation. Eq. (22) states that only one shift policy $p$ is to be implemented.

Table 5 presents the results for several tile production demand scenarios, ranging from a very small, $7000 \mathrm{~m}^{2}$, to a big demand, $70000 \mathrm{~m}^{2}$ of tile per week. Although a wider range of production rates can be selected, there are other factors that shall be taken into account in the decision making strategy, namely the logistic costs for the distribution of the ceramic tile product in the market. These kind of issues are beyond the scope of this work. These factors were taken into account to select a realistic range of ceramic
Table 5

Optimal solutions of the Problem II for various weekly ceramic tile productions, $P$

\begin{tabular}{rllllllc}
\hline$P / \mathrm{m}^{2}$ & M1 & M2 & M3 & M4 & Shift policy & $Z^{*} / €$ & CPU time $/ \mathrm{s}^{\mathrm{a}}$ \\
\hline 7000 & - & 1 & - & - & P1 & 1543.45 & 79.9 \\
14000 & 1 & - & - & - & P3 & 2500.44 & 61.7 \\
21000 & 1 & - & - & - & P3 & 2962.50 & 2295.6 \\
28000 & 1 & 1 & - & - & P3 & 4180.92 & 1691.2 \\
35000 & 2 & - & - & - & P3 & 4579.40 & 1369.6 \\
42000 & 2 & - & - & - & P3 & 5123.24 & 2478.5 \\
49000 & 2 & - & - & - & P3 & 5769.63 & 9031.6 \\
56000 & 2 & 1 & - & - & P3 & 6764.51 & 5313.3 \\
63000 & 3 & - & - & - & P3 & 7293.25 & $15470.6^{\mathrm{b}}$ \\
70000 & 3 & - & - & - & P3 & 7918.88 & 5172.1 \\
\hline
\end{tabular}

${ }^{\mathrm{a}}$ On a Windows XP Intel Core 2 Duo $1.83 \mathrm{GHz}$ computer.

${ }^{\mathrm{b}}$ The GAMS resource limit is hit when using the relative tolerance of $10^{-3}$. This solution is obtained when the relative tolerance is relaxed to $2 \times 10^{-3}$.

tile production demand with respect to the market context. Also, higher production rates lead to problems of higher dimensionality to reach feasible solutions, with the consequent increase in computational effort to solve them. To overcome this, other solution methodologies can be exploited to handle problems of extreme dimension, for instance genetic algorithms [24] and algorithms with heuristic approaches [25].

From Table 5, it follows that P3 is the optimal shift policy for a production higher than $7000 \mathrm{~m}^{2}$ per week. As mentioned previously, this shift policy provides $200 \mathrm{~h}$ of workforce, a number of hours superior to the $168 \mathrm{~h}$ of the production cycle. One emphasizes that the cost of increasing the workforce resources is lower than the depreciation cost of an additional grinding unit. Also, in the case of a production higher than $7000 \mathrm{~m}^{2}$ of ceramic tile per week, any optimal design solution standing on the installation of more than one unit is more cost effective with the shift policy P3 than a solution based on the quite limiting shift policy P1. For instance, for a weekly production of $42000 \mathrm{~m}^{2}$ there is need of only two mills of class M1 (total production cost of $€ 5123.24$, Table 5), whereas with shift policy P1 it requires 3 mills of class M1 and one mill of class M2 (total production cost of $€ 6464.73$, Table 4). Therefore, the adoption of shift policy P3 in this case leads to a cost reduction of about $21 \%$ with respect to the overall cost with shift policy P1. Shift policy P2 is never selected because to complete one sequence of feeding, grinding, and emptying tasks takes at least $15 \mathrm{~h}$, whereas the operators are only available for a period of time of $15 \mathrm{~h}$. Therefore, with shift policy P2 


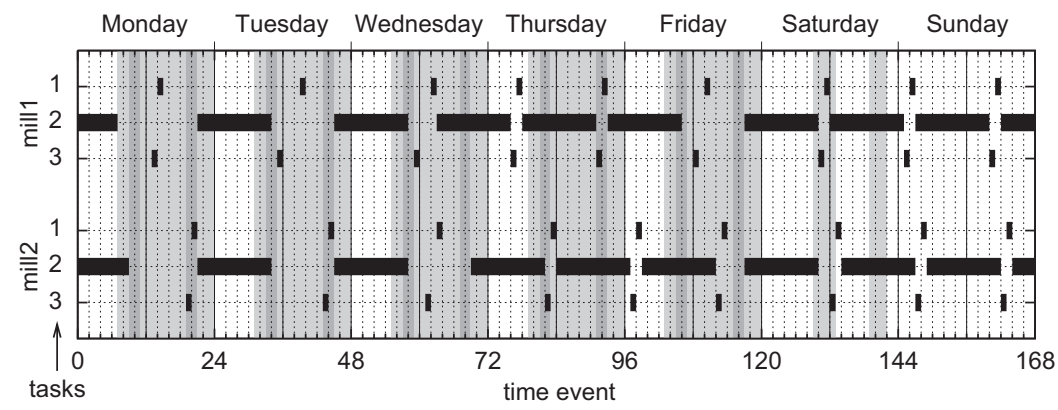

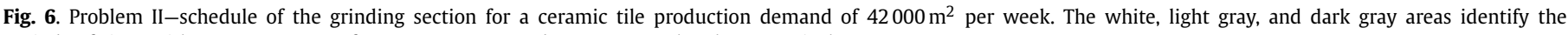
periods of time with an energy cost of $0.0481,0.0945$, and $€ 0.2161$ per $\mathrm{kW} \mathrm{h}$, respectively.

it is not possible to complete a batch cycle and to perform the load of the mill during periods of time such that one can take profit of overnight processing at reduced energy costs.

\subsection{Schedule for a tile production demand of $42000 \mathrm{~m}^{2}$ per week}

Fig. 6 presents the schedule of the grinding section for a ceramic tile production of $42000 \mathrm{~m}^{2}$ per week. Here, the optimal solution suggests the installation of only two ( $n=2$ in Fig. 2$)$ mills with the biggest net capacity available in the market $(i=1)$, mills of class M1 (Table 5). It is noteworthy to mention that the depreciation cost of the M1 mill units is $€ 216.89$ higher than the depreciation cost of the second largest mills (M2). Each batch from a M1 unit allows a production of $2389.7 \mathrm{~m}^{2}$ of ceramic tile, whereas the production rate that can be achieved with a M2 mill is of $1672.9 \mathrm{~m}^{2}$ per batch. Therefore the usage of lower capacity mills requires a higher number of batches to fulfill the production demand. A batch that is performed during the regular period of time, taking advantage of the lower price of the energy during the night period, costs on average $€ 129$. It turns out that the installation of a smaller unit would require two or more additional batches to satisfy the production goals. As demonstrated above, such solution is not suitable because the resulting overall energy cost overcomes the total depreciation cost savings from using smaller capacity equipment. This clearly explains why the optimal design solution is defined with M1 class units. Nevertheless, for the production rates of $35000 \mathrm{~m}^{2}$ and $42000 \mathrm{~m}^{2}$ of ceramic tile per week, both M1 units are operated in a sub-intensive way. Altogether, the 18 batches in Fig. 6 could satisfy a production demand of up to $43000 \mathrm{~m}^{2}$ of ceramic tile. Hence, the installed capacity may be used to target higher ceramic tile production demands $\left(49000 \mathrm{~m}^{2}\right.$ in Table 5 ). Another possible alternative configuration is the installation of one unit of class M1 and another of class M2 in order to decrease the total depreciation costs. As discussed above, this solution is not sufficiently cost effective to trade-off favorably with the consequent higher total energy cost.

Finally, a short note on the sequence of the last three batches of each mill processed during the weekend. One can observe in Fig. 6 that the batches of mill 2 are delayed $2 \mathrm{~h}$ with respect to the set of batches of mill 1 . This is because the problem is formulated such that no more than one workforce resource can be assigned to the same time slot. Otherwise, it would be possible to feed and empty the two mills at the same time, but that would require a more detailed description of the workforce plant activities besides the emptying and feeding tasks.

\section{Conclusions}

This work addresses a case study of the application of general frameworks devoted to simultaneous sizing and scheduling, such as the discrete-time RTN framework, to real problems commonly found in the ceramic industry sector. The case study comprises the optimal sizing of the grinding section of a ceramic tile plant. Because the cost of the resources required to operate the plant are of the same order as that of the equipment depreciation cost, the optimal sizing comprises the determination of the ceramic tile production schedule, the resources allocation, and the shift policy. The optimal scheduling of the grinding section is described by a discrete-time RTN process representation, using a fixed time horizon discretized into time slots, where states and resources availability changes. This subproblem is integrated into a general sizing problem, taking into consideration a set of resources (equipment, energy and workforce). To account for the resources availability specifications the initial point of the time horizon is fixed, and the resources availability constraints are defined accordingly. The resulting mathematical programming formulation has the form of a MILP model, and is solved by using the GAMS/CPLEX solver.

This approach was firstly applied considering that the workforce is constrained to a single operator, and later expanded to handle the decision regarding the selection of the best shift policy. The solution of the problem was successfully obtained for various ceramic tile production scenarios, with the corresponding optimal number of grinding units to install, their optimal schedule, and the most appropriate shift policy.

This example clearly illustrates the advantages of using optimization procedures to provide the decision-making with a quantitative and reproducible basis to design production units. Furthermore, it also highlights the need to determine the optimal operation mode, the scheduling of the associated production factors and policies before the plant installation, in order to achieve optimal performance, a rather important goal to keep a company in business.

\section{Acknowledgments}

The authors acknowledge the reviewers for their comments and suggestions that have helped to improve the contents and clarity of the manuscript.

\section{References}

[1] Grossmann I. Enterprise-wide optimization: a new frontier in process systems engineering. AIChE Journal 2005;51:1846-57.

[2] Méndez CA, Cerdá J, Grossmann IE, Harjunkoski I, Fahl M. State-of-the-art review of optimization methods for short-term scheduling of batch processes. Computers and Chemical Engineering 2006;30:913-46.

[3] Floudas CA, Lin X. Continuous-time versus discrete-time approaches for scheduling of chemical processes: a review. Computers and Chemical Engineering 2004;28:2109-29.

[4] Kallrath J. Planning and scheduling in the process industries. OR Spectrum 2002;24:219-50.

[5] Castro PM, Barbosa-Póvoa AP, Novais AQ. Simultaneous design and scheduling of multipurpose plants using resource task based continuous-time formulations. Industrial and Engineering Chemistry Research 2005;44:343-57.

[6] Yeh NC, Reklaitis GV. Synthesis and sizing of batch/semicontinuous processes. Computers and Chemical Engineering 1987;11:639-54. 
[7] Samsatli NJ, Shah N. Optimal integrated design of biochemical processes. Computers and Chemical Engineering 1996;S20:315-29.

[8] Voudouris VT, Grossmann IE. Mixed-integer linear programming reformulations for batch process design with discrete equipment sizes. Industrial and Engineering Chemistry Research 1992;31:1315-25.

[9] Kondili E, Pantelides CC, Sargent RWH. A general algorithm for short-term scheduling of batch operations-I. MILP formulation. Computers and Chemical Engineering 1993;17:211-27.

[10] Pantelides CC. Unified frameworks for optimal process planning and scheduling. In: Foundations of computer aided process operations. 1994. p. 253-74.

[11] Barbosa-Póvoa APFD, Macchietto S. Detailed design of multipurpose batch plants. Computers and Chemical Engineering 1994;18:1013-42.

[12] Barbosa-Póvoa APFD, Pantelides CC. Design of multipurpose production facilities: a RTN decomposition algorithm. Computers and Chemical Engineering 1999;S23:7-10.

[13] Lin X, Floudas CA. Design, synthesis and scheduling of multipurpose batch plants via an effective continuous-time formulation. Computers and Chemical Engineering 2001;25:665-74.

[14] Maravelias CT, Grossmann IE. Minimization of the makespan with a discretetime state-task network formulation. Industrial and Engineering Chemistry Research 2003;42:6252-7.

[15] Castro PM, Barbosa-Póvoa AP, Matos HA, Novais AQ. Simple continuous-time formulation for short-term scheduling of batch and continuous processes. Industrial and Engineering Chemistry Research 2004;43:105-18.

[16] Shah N, Pantelides CC, Sargent R. A general algorithm for short-term scheduling of batch operations-II. Computational issues. Computers and Chemical Engineering 1993;17:229-44.
[17] Shah N, Pantelides CC, Sargent RWH. Optimal periodic scheduling of multipurpose batch plants. Annals of Operations Research 1993;42:193-228.

[18] Schilling G, Pantelides CC. Optimal periodic scheduling of multipurpose plants. Computers and Chemical Engineering 1999;23:635-55.

[19] Castro PM, Barbosa-Póvoa AP, Matos HA. Optimal periodic scheduling of batch plants using RTN-based discrete and continuous-time formulations: a case study approach. Industrial and Engineering Chemistry Research 2003;42:3346-60.

[20] Pinto T, Barbosa-Póvoa APFD, Novais AQ. Optimal design and retrofit of batch plants with a periodic mode of operation. Computers and Chemical Engineering 2005;29:1293-303.

[21] Duque J, Barbosa-Póvoa APFD, Novais AQ. Synthesis and optimization of the recovery route for residual products under uncertain product demand. Computers and Operations Research 2007;34:1463-90.

[22] Turton R, Bailie RC, Whiting WC, Shaeiwitz JA. Analysis, synthesis and design of chemical processes. Englewood Cliffs, NJ: Prentice-Hall Inc.; 1998.

[23] Brooke A, Kendrick D, Meeraus A, Raman R. AMS-a users guide. Washington: GAMS Development Corporation; 1998.

[24] Wang C, Quan H, Xu X. Optimal design of multiproduct batch chemical process using genetic algorithms. Industrial and Engineering Chemistry Research 1996;35:3560-6.

[25] Luo Y, Guignard M, Chen C. A hybrid approach for integer programming combining genetic algorithms, linear programming and ordinal optimization. Journal of Intelligent Manufacturing 2001;12:509-19. 